\title{
Freki: Uma Ferramenta para Análise Automatizada de Malware
}

\author{
Cristian H. M. Souza ${ }^{1}$, Felipe S. Dantas Silva ${ }^{1}$ \\ ${ }^{1}$ LaTARC Research Lab \\ Instituto Federal de Educação, Ciência e Tecnologia do Rio Grande do Norte (IFRN) \\ Natal-RN, Brasil \\ cristianmsbregmail.com, felipe.dantaseifrn.edu.br
}

\begin{abstract}
Malware analysis is of utmost importance for information security. However, while many tools attempt to automate this process, few have a local installation option and a centralized environment for analyzing and exposing results. Furthermore, API query limitations and high license prices make these tools unattractive for newcomers to the field. This work proposes Freki, an open-source tool for automated analysis of malicious programs. The solution features a simple and intuitive interface in which users can submit files for analysis and promptly obtain the results obtained by the system. In addition, the proposed system delivers a REST API that allows new analyzes and queries to already investigated binaries via hash, allowing Freki to be the basis for the development of new applications for malware analysis.
\end{abstract}

Resumo. A análise de malware é de fundamental importância para a segurança da informação. Embora diversas ferramentas tentem automatizar esse processo, poucas apresentam uma opção de instalação local e um ambiente centralizado para análise e exposição dos resultados. Ademais, as limitações de consultas a APIs e os altos preços de licenças de uso tornam essas ferramentas pouco atrativas para iniciantes na área. Este trabalho propõe o Freki, uma ferramenta open-source para análise automatizada de programas maliciosos. A solução apresenta uma interface simples e intuitiva, na qual os usuários podem enviar arquivos para análise e obter prontamente os resultados obtidos pelo sistema. Além disso, a solução possui uma API REST que permite novas análises e consultas a binários já analisados via hash, permitindo que o Freki seja base para o desenvolvimento de novas aplicações para análise de malware.

\section{Introdução}

Infecções por malware continuam sendo uma das principais ameaças aos sistemas computacionais. Tais programas são responsáveis por causar prejuízos, através de alterações indevidas ou roubo de informações de usuários domésticos e empresas. Uma das formas eficazes de minimizar eventuais acessos não autorizados e, consequentemente, manter a integridade das informações é o uso da classificação via código hash [Kaur and Nagpal 2012]. Entretanto, essa técnica se torna ineficaz no combate de ameaças do tipo zero-day, sendo necessário recorrer a técnicas mais profundas para inspeção do binário, tais como a análise estática e a análise dinâmica [Gandotra et al. 2014].

Segundo um relatório publicado pela Malwarebytes [Malwarebytes 2020] em 2020, foi constatado um aumento de $13 \%$ nas detecções de malware focado no setor 
corporativo. O documento também mostra que atividades de ransomware estão sendo cada vez mais registradas. O relatório ainda destaca as frequentes infecções por $a d w a-$ res em dispositivos móveis. Este cenário exige o desenvolvimento de novos mecanismos de detecção, mitigação e análise dos diferentes tipos de malware desenvolvidos e aperfeiçoados dia após dia.

A análise de malware pode ser classificada em estática ou dinâmica, onde uma técnica complementa a outra [Chakkaravarthy et al. 2019]. Na análise estática, o usuário realiza a inspeção do binário (cabeçalhos, strings, capacidades, etc.), com o objetivo de definir sua função. Em contraste, na análise dinâmica, o usuário executa o binário em um ambiente controlado (máquina virtual ou sandbox), com o objetivo de monitorar seu comportamento (chamadas de sistema, consultas DNS, arquivos criados, etc.) [Aslan and Samet 2020].

Com o objetivo de dificultar a inspeção (p. ex. engenharia reversa), atacantes tem se empenhado cada vez mais em aperfeiçoar o uso de técnicas anti-análise. As principais são: (i) anti-disassembly (para evitar que o código assembly seja analisado corretamente); (ii) anti-debugging (para impedir a execução do programa em debuggers); e (iii) anti-VM (para ocultar o real comportamento do binário em máquinas virtuais) [Singh and Singh 2018].

Embora diversas iniciativas tenham sido desenvolvidas para fornecer suporte à análise de artefatos maliciosos, como o VirusTotal 1 e Any.Run ${ }^{2}$, nenhuma delas atualmente oferece uma forma de deploy local, na qual o usuário tem o total controle do binário a ser inspecionado. Vale notar que o arquivo (malicioso ou não) é muitas vezes compartilhado com terceiros, trazendo preocupações aos profissionais que precisam manter o sigilo e proteção dos dados analisados. Ademais, tais mecanismos possuem APIs limitadas, exigindo que o usuário adquira licenças de uso para consumir maiores quantidades de dados processados. Desse modo, é necessário o desenvolvimento de soluções capazes de criar melhores ambientes para a análise de malware.

Com base no exposto, este trabalho apresenta o Freki, uma nova ferramenta para análise automatizada de programas maliciosos. Freki é capaz de realizar rapidamente a análise estática de binários, fornecendo um relatório detalhado e compreensível aos usuários, além de permitir a posterior consulta e download dos resultados através de uma interface principal ou via API REST fornecida nativamente pelo sistema. Vale notar que a intenção não é substituir as ferramentas atuais, mas fornecer uma solução open-source, modular e de fácil implantação local.

O restante deste artigo está estruturado da seguinte forma: a Seção 2 apresenta a solução proposta e detalha sua arquitetura, enfatizando seus principais componentes; a Seção 3 contém as instruções para utilização do Freki e a demonstração planejada; a Seção 4 apresenta as considerações finais e delineia apontamentos de trabalhos futuros.

\section{Freki: Arquitetura e Implementação}

A solução proposta nesse trabalho é uma ferramenta open-source capaz de analisar estaticamente arquivos binários, com foco em programas maliciosos. O principal objetivo

\footnotetext{
${ }^{1}$ https://www.virustotal.com

${ }^{2}$ https://any.run
} 
é auxiliar os usuários na inspeção dos artefatos e averiguação das funcionalidades do arquivo. A análise permite a identificação de diversas características do binário, tais como: hashes, avaliação por antivírus, arquitetura, cabeçalhos, seções, importações e arquivos ocultos. Esses resultados podem orientar os usuários nas análises dinâmicas e auxiliar na classificação das diversas famílias de malware.

\subsection{Arquitetura}

A arquitetura do Freki é composta por diferentes módulos especializados em uma etapa da análise dos artefatos. Freki disponibiliza uma interface única e de fácil utilização para análise de binários, além de permitir a criação de relatórios e a posterior consulta a arquivos já analisados via seu hash SHA-1. A Figura 1 apresenta uma visão geral da arquitetura do Freki e seus módulos funcionais. Uma das principais vantagens da ferramenta é a sua simplicidade, além da fácil instalação, viabilizada através de recursos de conteinerização via Docker ${ }^{3}$.

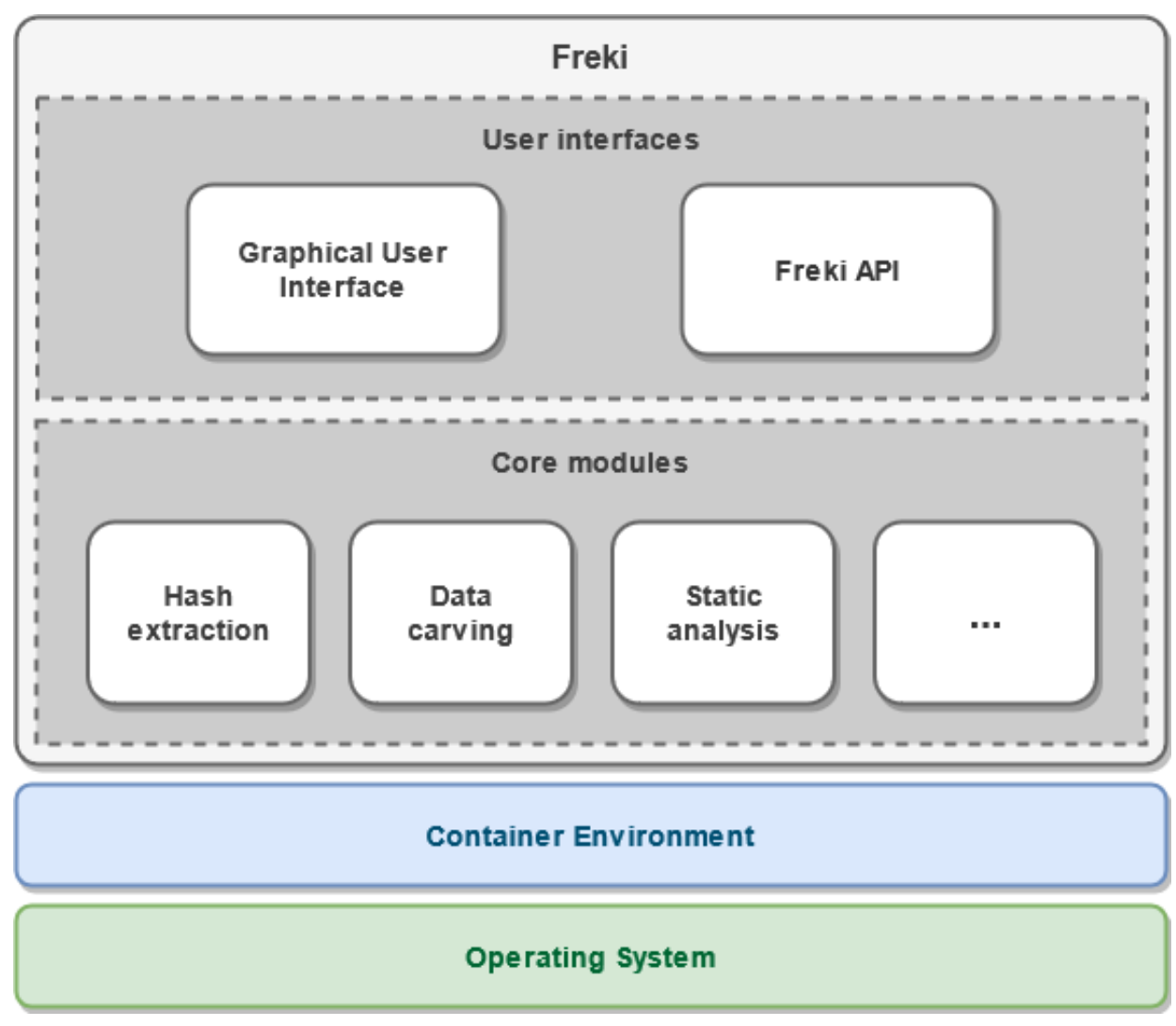

Figura 1. Arquitetura do Freki.

A ferramenta consiste em três componentes principais: (i) user interfaces, onde é possível utilizar uma plataforma web para envio e consulta dos arquivos analisados ou realizar solicitações à sua API REST via linha de comando; (ii) core modules, local das funcionalidades principais do sistema e onde todas as análises são orquestradas; e (iii) container environment, que garante o isolamento, fácil instalação e gerenciamento da aplicação.

\footnotetext{
${ }^{3}$ https://www.docker.com/
} 
Como ilustrado na Figura 2, o ambiente virtualizado através dos containers é composto por três elementos: (i) servidor web Nginx $]^{4}$ atuando como proxy reverso para a aplicação principal; (ii) a própria aplicação, que se comunica com os demais containers para envio e armazenamento de dados; e (iii) instância de um banco de dados relacional (implementado através do MariaDB ${ }^{5}$ ), responsável por armazenar os resultados das análises e responder as solicitações da aplicação.

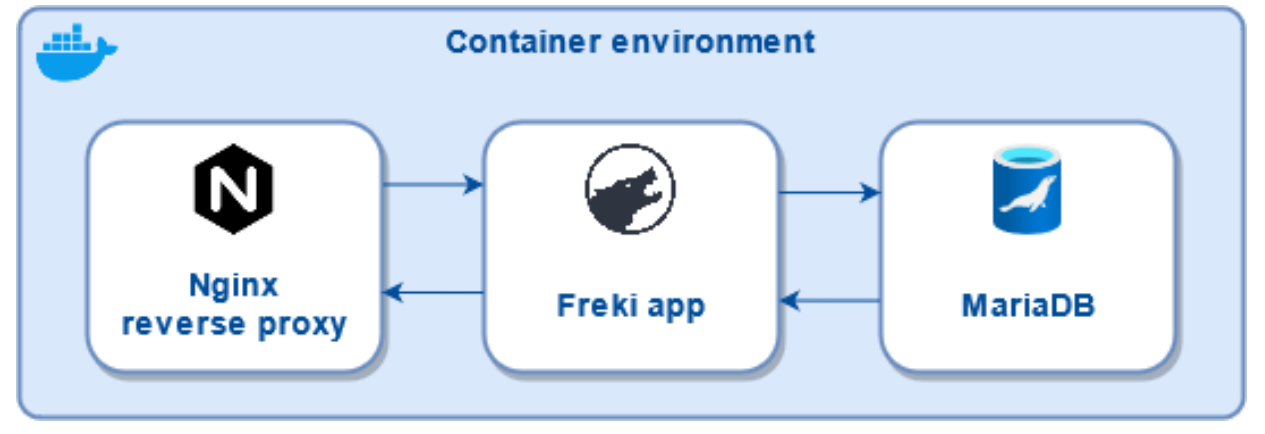

Figura 2. Ambiente de containers do Freki.

As subseções a seguir descrevem os principais componentes do Freki, seus módulos e funcionalidades. Vale notar que a a arquitetura modular do Freki foi planejada para viabilizar a fácil manutenção e inclusão de novos módulos pelos seus usuários, elevando seu potencial de análises e garantindo resultados coerentes para diferentes tipos de arquivos.

\subsubsection{User Interface (UI)}

Freki apresenta uma interface simples e autocontida, na qual os usuários podem facilmente navegar entre os principais recursos disponíveis. A página principal apresenta aos usuários os últimos arquivos enviados para análise, bem como permite o acesso a estes dados para armazenamento local. Após o envio do binário a ser analisado, o usuário é redirecionado para a página de análise exclusiva de cada arquivo, que contém detalhes e características do programa inspecionado - como exposto na Figura 3 . Por questões de segurança, é necessário criar uma conta na plataforma para o envio de arquivos. Entretanto, a navegação e descarregamento dos arquivos podem ser realizadas por qualquer usuário. Vale notar que todos os arquivos disponibilizados para download são compactados e protegidos por senha. Ademais, a aplicação permite que os usuários autenticados façam comentários, permitindo uma melhor comunicação entre os usuários a respeito dos resultados das análises.

\subsubsection{REST API}

Visando expandir a usabilidade da ferramenta, uma API REST foi desenvolvida e integrada à aplicação. Desse modo, é possível realizar consultas ao Freki através de diferentes ferramentas e linguagens de programação. Logo, novos mecanismos para análise

\footnotetext{
${ }^{4}$ https://www.nginx.com

${ }^{5}$ https://mariadb.org
} 


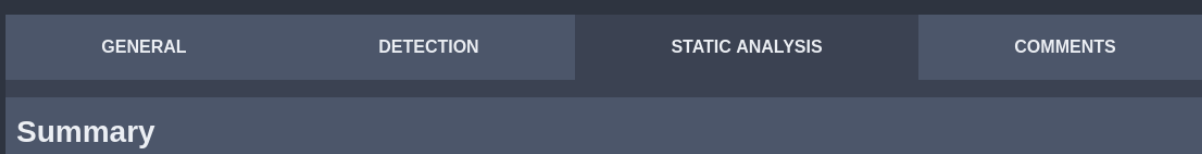

Summary

Figura 3. Interface principal do Freki.

de malware podem consultar diretamente o Freki e utilizar os dados fornecidos internamente para os mais diferentes propósitos, como resposta a incidentes e análise forense. Visando dificultar ataques de negação de serviço, por padrão, há um limite de 4 requisições por segundo à API. Porém, esse valor pode ser customizado pelos proprietários das instâncias do Freki dependendo da necessidade. Toda a definição, criação e documentação da API foi realizada através do framework Swagger

\subsubsection{Core Modules}

O core do Freki é o componente responsável por concentrar as principais funcionalidades utilizadas durante a execução da aplicação. Esse componente contém os módulos que atualmente abrangem as seguintes funções: (i) extração dos hashes do arquivo; (ii) consultas à plataforma VirusTotal; (iii) análise estática; (iv) data carving; e (v) classificação do binário. Uma breve descrição de cada funcionalidade é fornecida a seguir:

- Extração dos hashes: são calculados vários hashes com o objetivo de facilitar a identificação do binário dentro do Freki e na Internet. Até o momento, os seguintes hashes são suportados: MD5, SHA-1, SHA-256, SHA-384, SHA-512, CRC32 e SSDEEP;

\footnotetext{
${ }^{6} \mathrm{https}: / /$ swagger.io
} 
- Consultas ao VirusTotal: a plataforma VirusTotal fornece uma API de fácil utilização para o envio de binários a diferentes antivírus (p. ex. Avast, Avira, ClamAV e Kaspersky). Desse modo, é possível garantir um maior nível de acurácia na identificação de um arquivo malicioso. Caso o usuário forneça a sua chave de API do VirusTotal, o Freki é capaz de realizar consultas automáticas a essa API e apresentar os dados fornecidos pelos diferentes motores. Caso contrário, o administrador também pode fornecer uma chave mestra que será utilizada para consultar os arquivos enviados por todos os usuários da instância;

- Análise estática: a análise estática é uma etapa essencial para identificação de várias características do executável. Diante disso, Freki é capaz de extrair diversas informações a respeito do arquivo em questão, tais como arquitetura, cabeçalhos, seções, importações, capacidades e strings;

- Data carving: muitos programas maliciosos possuem arquivos ocultos que necessitam de técnicas especiais para extração. Logo, Freki provê um mecanismo para data carving, possibilitando a extração dos artefatos antes da execução do binário;

- Classificação do binário: visando auxiliar usuários e pesquisadores na identificação e classificação dos arquivos analisados, a aplicação é capaz de identificar características e famílias de malware através de regras de classificação predefinidas.

\subsection{Implementação}

O back-end do Freki é totalmente implementado na linguagem Python, fazendo uso das seguintes bibliotecas que automatizam o trabalho de implementação: o framework web utilizado foi o Flask [Pallets 2010], devido a sua grande modularidade e simplicidade, juntamente com o servidor Guni corn [Chesneau 2009]. Para acesso ao banco, foi utilizado o ORM (Object Relational Mapper) SQLA lchemy [SQLAlchemy 2018]. O parsing das informações contidas em executáveis PE (Portable Executable) é realizado através da biblioteca pefile [Carrera 2015] e a identificação das suas capacidades é realizada através do capa [FireEye 2020]. Já o processo de data carving é realizado pelo Foremost [Kris Kendall and Mikus 2001]. Por fim, a classificação dos artefatos é realizada através do Yara [VirusTotal 2012] e mais um conjunto predefinido de regras fornecidas pela comunidade ${ }^{7}$. O front-end da ferramenta foi construído através do framework Bootstrap ${ }^{8}$ garantindo um fácil desenvolvimento de design responsivo dos componentes em diferentes dispositivos.

O ambiente base para execução do Freki é operacionalizado sob a plataforma Docker. A orquestração do ambiente foi estruturada através de múltiplos Dockerfiles, criados para instruir ao Docker Compose $^{9}$ os passos necessários para o correto funcionamento da aplicação. Além disso, uma rede privada exclusiva para o Freki é criada ao

\footnotetext{
${ }^{7}$ https://github.com/Yara-Rules/rules

${ }^{8}$ https://getbootstrap.com

${ }^{9}$ https://docs.docker.com/compose
} 
iniciar a aplicação, expondo apenas as portas essenciais para o acesso por parte do usuário e garantindo um maior isolamento do ambiente.

\section{Experimentação}

Maiores detalhes sobre o Freki, como o código-fonte, funcionamento básico e manuais para instalação e utilização (além de um vídeo da ferramenta em funcionamento) podem ser encontrados no repositório oficial do projeto ${ }^{10}$. É possível realizar a instalação da ferramenta de forma nativa, sem a necessidade do Docker. No entanto, o processo de instalação simplificado é descrito a seguir:

1. Acesso ao código-fonte: git clone https://github.com/crhenr/ freki.git;

2. Alteração do arquivo .env: este arquivo permite a configuração das credenciais desejadas (é recomendado o uso de uma boa política de senhas);

3. Configuração do HTTPS: é recomendável habilitar o protocolo HTTPS (principalmente se a instância estiver diretamente exposta à Internet). Para isso, é necessário adicionar o certificado e a chave privada no diretório docker/nginx/certs;

4. Execução do Freki: make run ou docker-compose up -d.

\subsection{Demonstração}

O código-fonte, documentação e instruções para instalação via Docker ou nativa estão disponíveis em https://github.com/crhenr/freki. O plano para demonstração do Freki no salão de ferramentas é apresentar o comportamento da solução diante da análise automatizada de exemplares de malware reais, proporcionando aos conferencistas uma visão geral do processo de análise de programas maliciosos e como o Freki pode facilitar esse processo. Também será apresentado o processo de utilização da API do Freki em diferentes cenários, evidenciando a extensibilidade da ferramenta. Uma demonstração em vídeo da ferramenta pode ser encontrada em: https: //youtu.be/brvNUPgw7ho.

\section{Considerações finais e trabalhos futuros}

A análise de malware é fundamental para a segurança da informação. Conforme as famílias de programas maliciosos evoluem, novas ferramentas se fazem necessárias para detectar, mitigar e analisar tais ameaças. Neste trabalho propomos o Freki, uma ferramenta para análise automatizada de malware. A arquitetura da solução fornece um ambiente completo para análise de programas maliciosos de forma simples e intuitiva. Além disso, é possível estender as funcionalidades da aplicação através de customizações no código-fonte ou utilizando a sua API.

Como trabalhos futuros, pretendemos adicionar o suporte a uma quantidade maior de tipos de arquivos (p. ex. ELF, PDF, APK, etc.), visando auxiliar os usuários na

\footnotetext{
${ }^{10}$ https://github.com/crhenr/freki
} 
identificação de ameaças para diferentes plataformas, incluindo dispositivos habilitados para o cenário da Internet das Coisas (IoT, do inglês Internet of Things). Também serão implementados recursos de análise dinâmica dos artefatos em diferentes plataformas através de máquinas virtuais (VMs, do inglês Virtual Machines) especialmente projetadas para essa finalidade. Ademais, pretendemos aperfeiçoar ainda mais o código-fonte e a arquitetura do Freki, garantindo o maior desempenho possível nas análises.

\section{Agradecimentos}

Os autores agradecem ao Conselho Nacional de Desenvolvimento Científico e Tecnológico (CNPq) e ao Instituto Federal de Educação, Ciência e Tecnologia do Rio Grande do Norte (IFRN) pelo apoio ao desenvolvimento desse trabalho.

\section{Referências}

Aslan, Ö. A. and Samet, R. (2020). A comprehensive review on malware detection approaches. IEEE Access, 8:6249-6271.

Carrera, E. (2015). pefile. https://github.com/erocarrera/pefile.

Chakkaravarthy, S. S., Sangeetha, D., and Vaidehi, V. (2019). A survey on malware analysis and mitigation techniques. Computer Science Review, 32:1-23.

Chesneau, B. (2009). Gunicorn wsgi http server for unix. https: //gunicorn . org/.

FireEye (2020). capa. https: / / github. com/fireeye/capa.

Gandotra, E., Bansal, D., and Sofat, S. (2014). Malware analysis and classification: A survey. Journal of Information Security, 2014.

Kaur, G. and Nagpal, B. (2012). Malware analysis \& its application to digital forensic. International Journal on Computer Science and Engineering (IJCSE), 4(04):622-626.

Kris Kendall, J. K. and Mikus, N. (2001). Foremost. http://foremost. sourceforge.net.

Malwarebytes (2020). 2020 state of malware report. https:// www.malwarebytes.com/resources/files/2020/02/2020_ state-of-malware-report-1.pdf

Pallets (2010). Flask documentation. https: / / flask.palletsprojects.com/ en $/ 2.0 . x /$

Singh, J. and Singh, J. (2018). Challenge of malware analysis: malware obfuscation techniques. International Journal of Information Security Science, 7(3):100-110.

SQLAlchemy (2018). Sqlalchemy - the database toolkit for python. https://www. sqlalchemy.org/.

VirusTotal (2012). Yara. https://github.com/VirusTotal/yara. 